\title{
Aortic valve reconstruction in myxomatous degeneration of aortic valves: Are fenestrations a risk factor for repair failure?
}

\author{
Hans-Joachim Schäfers, MD, ${ }^{\mathrm{a}}$ Frank Langer, MD, ${ }^{\mathrm{a}}$ Petra Glombitza, MD, ${ }^{\mathrm{a}}$ Takashi Kunihara, MD, ${ }^{\mathrm{a}}$ \\ Roland Fries, $\mathrm{MD},{ }^{\mathrm{b}}$ and Diana Aicher, $\mathrm{MD}^{\mathrm{a}}$
}

\begin{abstract}
Objective: Aortic valve repair is a more recent approach for the treatment of aortic regurgitation. Limited data exist for reconstruction in specific pathologies with isolated cusp pathology. We analyzed the results of aortic valve repair in patients with aortic regurgitation caused by myxomatous cusp prolapse in the presence of tricuspid valve anatomy and normal root size.
\end{abstract}

\begin{abstract}
Methods: Over a 12-year period, 111 patients underwent aortic valve reconstruction for regurgitant tricuspid aortic valves without concomitant root dilatation. Cusp prolapse was caused by myxomatous degeneration in 72 subjects (group I) and associated with fenestrations in 39 subjects (group II). Prolapse was corrected by means of plication of the free margin in the presence of normal cusp tissue only $(n=62)$ or combined with triangular resection of cusp tissue $(n=10)$. It was treated with additional closure of the fenestration with autologous pericardium in 39 instances (group II). Follow-up was complete in 98.5\% (cumulative 385 years).
\end{abstract}

Results: Hospital mortality was $1.8 \%$, and during follow-up, there was 1 thromboembolic event and no endocarditis. Freedom from reoperation at 5 and 8 years was $96 \%$.

Conclusions: Isolated cusp prolapse is a relevant cause of aortic regurgitation in tricuspid aortic valves without concomitant root dilatation. In myxomatous stretching of cusp tissue, plication of the free margin suffices to restore cusp geometry and aortic valve function. In the presence of fenestrations, reconstruction of normal cusp configuration can be achieved by means of closure of the fenestration with a pericardial patch. The midterm stability of both approaches is good. (J Thorac Cardiovasc Surg 2010;139:660-4)

Aortic valve replacement is an established treatment for patients with severe aortic regurgitation (AR), ${ }^{1}$ but it exposes the patient to the typical prosthesis-related complications. Both mechanical and biologic valve prostheses have been shown to be associated with an incidence of valve-related complications of $4 \%$ to $5 \%$ per patient-year. ${ }^{2-4}$

Reconstructive surgery of the aortic valve is becoming an attractive alternative to replacement. Apart from the advantage of not requiring anticoagulation, we have seen few valve-related complications after aortic valve repair. ${ }^{5}$ Thus far, however, reconstruction plays a limited role in the treatment of AR. This is because AR is caused by different valve pathologies and because there is a lack of standardized techniques for specific abnormalities. A more widespread use of aortic valve repair will require the definition of typical valve pathologies and the development of standardized techniques with predictable outcomes for their management.

\footnotetext{
From the Department of Thoracic and Cardiovascular Surgery, ${ }^{\mathrm{a}}$ University Hospitals of Saarland, Homburg/Saar, Germany, and Gotthard-Schettler-Klinik, ${ }^{\text {b }}$ Bad Schönborn, Germany.

Disclosures: Name.

Received for publication Dec 25, 2008; revisions received March 26, 2009; accepted for publication June 23, 2009; available ahead of print Aug 19, 2009.

Address for reprints: Hans-Joachim Schäfers, MD, Department of Thoracic and Cardiovascular Surgery, University Hospitals of Saarland, 66421 Homburg/Saar, Germany (E-mail: h-j.schaefers@uniklinikum-saarland.de).

$0022-5223 / \$ 36.00$

Copyright (c) 2010 by The American Association for Thoracic Surgery doi:10.1016/j.jtcvs.2009.06.025
}

Accepted techniques have been developed for aortic dilatation. Cusp repair approaches have been described for regurgitant bicuspid aortic valves. ${ }^{6,7}$ Cusp prolapse is also a reason for $\mathrm{AR}^{8-11}$ in tricuspid aortic valve anatomy and is assumed to be caused by myxomatous degeneration. It has been suggested that myxomatous degeneration is the primary cause for AR in $10 \%$ to $36 \%$ of patients with pure AR. ${ }^{10,11}$ Prolapse can also occur in conjunction with congenital fenestrations, which are typically located in the paracommissural area of the cusps. ${ }^{12-15}$ These fenestrations have been found in aortic valves that exhibited the features of myxomatous degeneration. ${ }^{8,14}$ The fenestrations are located in the coaptation zone of the cusps and thus do not directly result in regurgitation. The free cusp margin consists only of a thin fibrous strand, and elongation or rupture of the strand will lead to distortion of the cusp and consequent prolapse and regurgitation. ${ }^{12-15}$ The involved cusps are usually pliable and thus potentially suitable for repair. On the other hand, the type of defect and the thinness of the tissue might lead to concerns of increased risk of failure.

Cusp prolapse in tricuspid aortic valves has been treated with plication of the free cusp margin, ${ }^{5}$ triangular resection of redundant cusp tissue, ${ }^{16}$ or placement of a continuous polytetrafluoroethylene (PTFE) suture into the free margin. ${ }^{17}$ Based on stress distribution in the cusp, we have consistently preferred plication of the margin in its central portion. ${ }^{5,18} \mathrm{At}$ this time, there are only a few reports dealing with isolated correction of cusp prolapse, and there are no data dealing 


\section{Abbreviations and Acronyms \\ $\mathrm{AR}=$ aortic regurgitation \\ PTFE $=$ polytetrafluoroethylene}

with this specific subset of tricuspid aortic valves and normal root size.

Fenestrated cusps are seemingly an even bigger challenge for repair. The tissue quality of the cusps makes it more difficult to handle with sutures. Prolapse is not symmetric, and triangular resection or plication of the free margin does not correct the pathology. A PTFE suture placed as replacement of the marginal strand will be exposed to high stress, and a suture on the margin of the remaining cusp tissue will not restore normal cusp dimensions. Although PTFE has been used, ${ }^{17}$ no functional data have been published yet.

We have routinely corrected cusp prolapse in tricuspid aortic valves using consistent techniques. In the presence of thinned but otherwise normal cusp tissue, prolapse was corrected by means of central plication of the free margin. Triangular resection of cusp tissue was performed only for excessive cusp redundancy. If prolapse was caused by elongation or rupture of the marginal strand in a fenestrated cusp, the involved fenestration was routinely closed with a patch of autologous pericardium. Central plicating sutures were added in the free margin as needed.

The purpose of this report was to analyze the repair results for myxomatous degeneration and to compare the results with those obtained in fenestrated cusps.

\section{MATERIALS AND METHODS}

Between October 1995 and May 2008, 1013 patients were treated for cardiovascular disease involving AR. The valve was reconstructed in 826 instances. For the present analysis, only patients with tricuspid aortic valve morphology and absence of aortic dilatation were included, and follow-up was complete to November 2008. Active or healed endocarditis was excluded. Of all subjects, 114 fulfilled the morphologic criteria, 111 of whom underwent repair (age, 1-85 years; mean age, $57 \pm 17$ years; male/female ratio, 85/26). The aortic valve was replaced in 3 patients because of intraoperative conversion $(n=2)$ or the presence of fenestrations with resulting cusp deformation in all 3 cusps $(n=1)$. These latter patients were not analyzed further.

In the 111 repaired aortic valves, preoperative AR was severe in 82 and moderate in 29 instances. Cusp prolapse caused by degeneration with otherwise preserved cusp morphology was found in 72 instances, and in 39 fenestrations with elongated or ruptured marginal strands were present. The presence of a fenestration was difficult to predict preoperatively. Prolapse was most frequent in the right coronary cusp $(n=90)$, followed by the noncoronary $(n=63)$ and left coronary $(n=18)$ cusps. In these 111 patients isolated AR without need for concomitant surgical intervention was present in 39 instances. In the majority AR was found in combination with coronary artery disease $(\mathrm{n}=35)$ or other cardiac diseases (mitral regurgitation, $\mathrm{n}=$ 35 ; permanent atrial fibrillation, $\mathrm{n}=21$; or persistent foramen ovale, $\mathrm{n}=$ 6)). AR was the primary indication for surgical intervention in 82 subjects.

The repair strategy was constant in all cases. Aortic root dimensions were measured by means of transesophageal echocardiographic analysis before extracorporeal circulation. Aortoventricular diameter ranged from 25 to
$29 \mathrm{~mm}$ (mean, $27 \mathrm{~mm}$ ), sinotubular diameters ranged from 25 to $30 \mathrm{~mm}$ (mean, $28.5 \mathrm{~mm}$ ), and sinus diameter was less than $40 \mathrm{~mm}$ (mean, 35 $\mathrm{mm}$ ). After transverse aortotomy and induction of cardioplegia, stay sutures were placed on the 3 commissures and kept under tension throughout the procedure. The geometry of the cusps was assessed by means of direct measurement of geometric height (from the nadir of the sinus to the free edge with the cusp stretched) and effective height. ${ }^{19,20}$ Geometric height in normal (ie, nonrestricted) cusps ranged from 17 to $22 \mathrm{~mm}$ (mean, $19.7 \mathrm{~mm}$ ). Effective height in normal cusps ranged from 9 to $10 \mathrm{~mm}$ (mean, $9.3 \mathrm{~mm}$ ).

Cusp prolapse was defined as published previously. ${ }^{21}$ Three stay sutures (5-0 or 6-0 Prolene sutures; Ethicon, Hamburg, Germany) were placed in the corresponding free cusp margins at equal distance from the commissures, taking care to place them close to the nodulus of Arantius (ie, the central portion of the free margin; Figure 1). If prolapse was confined to 1 cusp only $(\mathrm{n}=56)$, the distance between the 2 stay sutures of the affected free cusp margin indicated the excess of length to be corrected (Figure 1). The free margin was then shortened with simple interrupted 5-0 or 6-0 polypropylene sutures (Prolene sutures, Figure 1). After each suture, the effect on cusp geometry was assessed, and additional sutures were placed as needed. If prolapse was present on $2(n=44)$ or $3(n=11)$ cusps, the first 2 cusps were repaired by shortening of the free margin until an effective height of 9 to $10 \mathrm{~mm}$ was reached. The third cusp was then corrected as described above. Plication of the free margin was performed most frequently on the right $(\mathrm{n}=52)$ and noncoronary $(\mathrm{n}=54)$ cusps and less frequently on the left cusp $(n=14)$. If marked redundancy of cusp tissue was present, a triangular resection was performed in the central portion of the cusp $(n=10)$. Correction of prolapse was considered adequate if all 3 free margins were at identical height and if the effective height was $9 \mathrm{~mm}$.

In the presence of fenestrations, the approach was modified. A fenestration was only addressed surgically if prolapse was present. A cusp stay suture for the cusp affected by the fenestration was placed in the central portion of the involved and adjacent cusps. Prolapse correction was intended to include closure of the fenestration; that is, implantation of the patch was meant to correct any additional distortion of cusp tissue.

A glutaraldehyde-fixed patch of autologous pericardium was prepared. The patch was intentionally made $10 \%$ to $20 \%$ larger than the size of the fenestration to avoid cusp restriction. With the cusp stay suture kept under tension, the patch was then sutured into the fenestration up to the commissure by using a running circumferential $6-0$ polypropylene suture (Figure 2 ). The suture was locked several times to avoid restriction from excessive tension on the suture line. The resulting cusp configuration was then assessed. Residual prolapse was corrected by using single plicating sutures on the central free margin. A fenestration was closed most frequently on the right cusp $(n=28)$ and less frequently on the left $(n=4)$ or noncoronary $(n=9)$ cusps. Two fenestrations were closed in 2 individuals.

Subcommissural root plication ${ }^{22}$ was performed in $89(80 \%)$ operations for additional stabilization of the repair by using braided sutures and Teflon pledgets (3-0 Ethibond, Ethicon) mostly confined to the commissures of the repaired cusp. In 87 instances a suture was placed beneath the right/noncoronary commissure, in 63 beneath the left/noncoronary commissure, and in 59 beneath the right/left commissure. Postoperatively, all patients were given aspirin $(100 \mathrm{mg} / \mathrm{d})$ for 2 months.

Intraoperatively, all patients were studied with transesophageal echocardiographic analysis (Siemens Sequoia; Siemens, Erlangen, Germany). The degree of AR was determined according to current recommendations. ${ }^{23}$ Further echocardiographic studies were performed with transthoracic echocardiography. All patients were studied between postoperative days 5 and 8 . Further follow-up studies were performed after 3, 6, and 12 months and thereafter at yearly intervals. All patients were followed, and a careful history was taken for clinical evidence of embolic events, bleeding complications, and other possible valve-related problems. Follow-up was complete for $98.5 \%$ of the patients. The follow-up ranged from 5 to 141 months, with a mean of $2.9 \pm 2.2$ years. Cumulative follow-up was 4624 patientmonths (mean, $35 \pm 26$ months). 


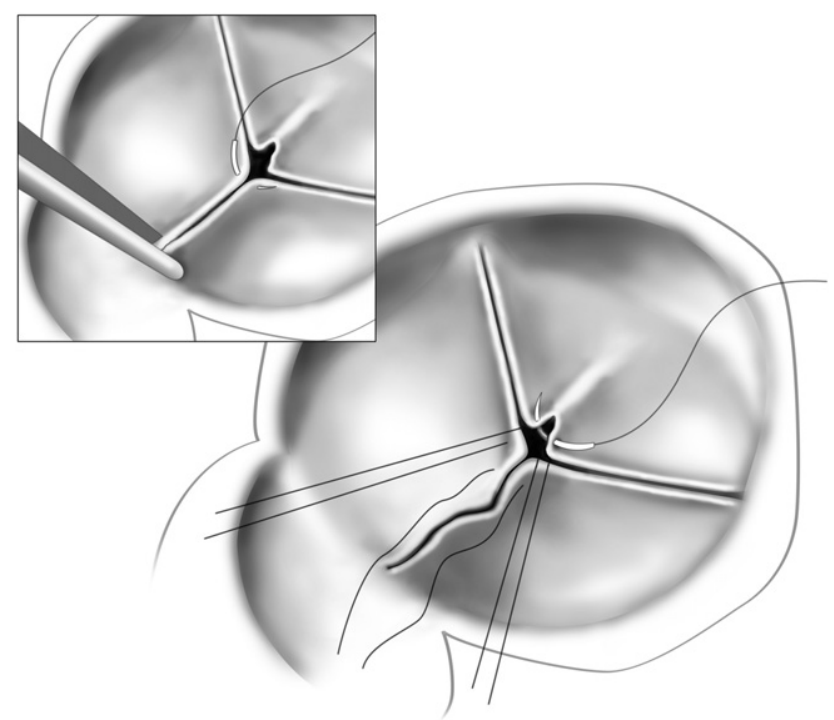

FIGURE 1. Cusp stay sutures are helpful to assess the relative length of the free margins. Two adjacent free margins are held under tension, and a 5-0 suture is placed through the free margin at corresponding points. This stay suture is ideally placed in the center of the free margin (ie, through the noduli of Arantius). Cusp stay sutures have been placed through the free margins in all 3 coaptation lines. Because the distance from the commissure is identical for all 3 sutures, the distance between 2 stay sutures on the free margin of the right coronary cusp indicates the degree of tissue redundancy. This is then plicated with 1 or several interrupted sutures.

For further analysis, the patients were divided into 2 groups. In group I cusp prolapse was corrected by means of plication of the free margin only. In group II cusp repair involved closure of a fenestration. AR ranged from moderate $(n=35)$ to severe $(n=37)$ in group I compared with a higher proportion of severe AR in group II (moderate, $n=5$; severe, $n=34$ ). The proportion of patients undergoing isolated valve repair was similarly higher in group II.

In group I 1 cusp was involved in 39 patients, 2 cusps were involved in 29 patients, and 3 cusps were involved in 4 patients. In group II 1 cusp was prolapsing in 16 patients, 2 cusps were prolapsing in 15 patients, and 3 cusps were prolapsing in 8 patients. In that group fenestrations were corrected in 1 $(\mathrm{n}=31)$ or 2 cusps $(\mathrm{n}=8)$.

Continuous data are presented as means \pm standard deviations. Statistical analysis included comparison of parametric and continuous variables between groups by using 1-way analysis of variance. Kaplan-Meier curves were calculated for freedom of relevant regurgitation, freedom from reoperation, freedom from valve replacement, and freedom from valve-related complications by using a commercially available software package (Prism; GraphPad, Inc, San Diego, Calif).

\section{RESULTS}

Hospital mortality was $1.8 \%$ (2/111 patients). No patient died after isolated aortic valve repair. The 2 patients with fatal outcomes presented with a combination of AR and coronary triple-vessel disease with decreased left ventricular function. The cause of death was mesenteric ischemia in both patients.

Postoperative morbidity was low. No patient had to be reexplored for bleeding, and there was no postoperative atrio-

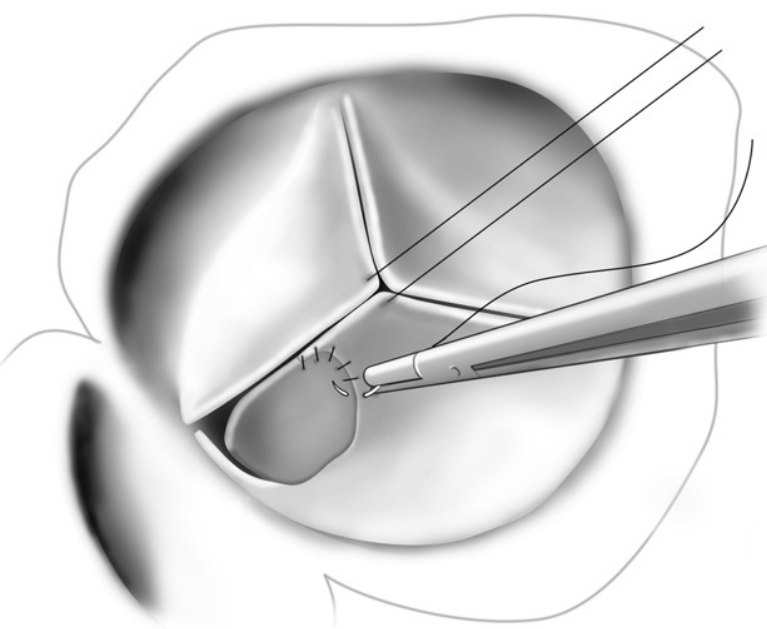

FIGURE 2. Drawing of repair of a cusp fenestration. The cusp is kept under tension, with the stay suture placed through its nodulus of Arantius and that of the neighboring cusp. A pericardial patch is inserted into the fenestration with a circumferential suture.

ventricular block. In all patients aortic valve function was improved compared with preoperative status; mild regurgitation was present 17 patients and was minimal $(n=58)$ or absent $(\mathrm{n}=36)$ in the remaining 94 patients. The proportion of patients with no or trivial regurgitation was significantly lower in group I (43/72 [60\%]) than in group II (31/39 [80\%], $P=.035)$. The preoperative degree of AR had no influence on postoperative AR $(P=.7)$.

Aortic valve function remained stable in the majority of patients throughout the follow-up period. Reoperation was necessary for recurrent regurgitation in 4 patients between 3 and 19 months postoperatively. The reasons for reoperation were cusp retraction unrecognized at the initial intervention in 1 patient ( 3 months) and recurrent prolapse in another patient (15 months). Mild-to-moderate AR was seen postoperatively in both individuals, and this increased over time. One patient had secondary root dilatation with increasing aortic insufficiency and required composite replacement of the valve and aorta 19 months postoperatively. In the patient from group II who required reoperation, a tear was found between the cusp tissue and the implanted pericardial patch. This was rerepaired, including the addition of a continuous PTFE suture to the free cusp margin.

Postoperative endocarditis did not occur. There was 1 thromboembolic complication: transient ischemic attack in conjunction with paroxysmal atrial fibrillation. Freedom from valve-related complications was $93 \%$ in group I and $97 \%$ in group II at 5 and 8 years postoperatively.

At 5 and 8 years, freedom from AR of class II or greater was $77 \%$ in the whole population. At 5 years, no patient had moderate regurgitation, except for the patients undergoing reoperation. There was no difference between the 2 groups, with $79 \%$ for plication of the margin and $71 \%$ for 


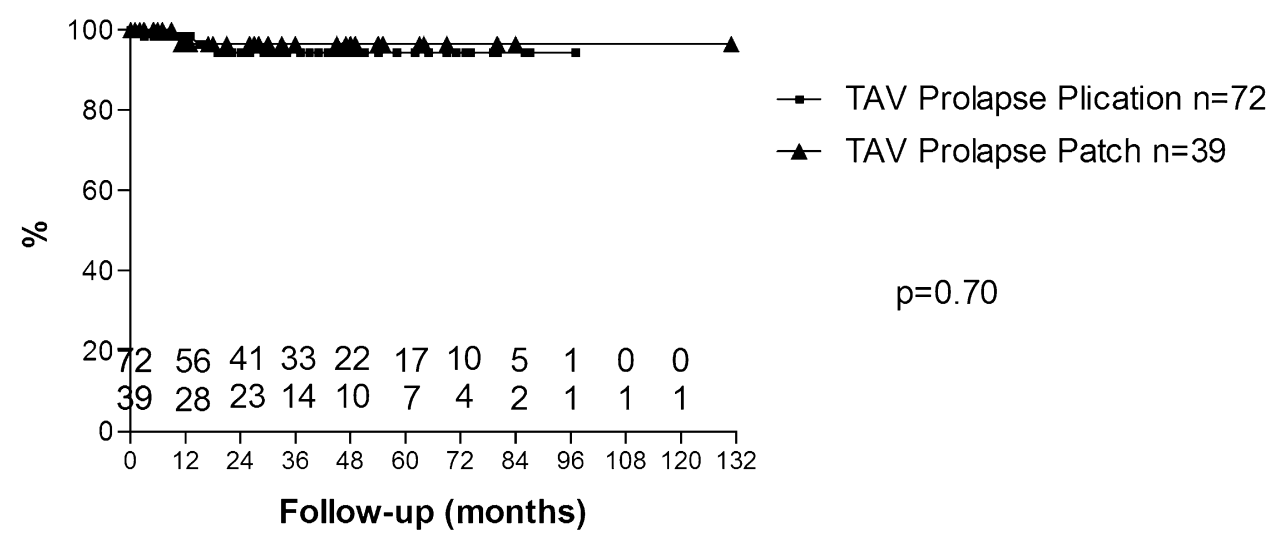

FIGURE 3. Freedom from reoperation after aortic valve repair for regurgitant tricuspid aortic valves (TAV).

repaired fenestrations $(P=.32)$. Freedom from reoperation at 5 and 8 years was $95 \%$ for all patients, $94 \%$ in group I, and $97 \%$ in group II $(P=.7$, Figure 3$)$. For patients with 1 repaired cusp, freedom from reoperation at 5 years was $95 \%$ compared with $94 \%$ after repair of 2 cusps and $100 \%$ after correction of 3 cusps $(P=.78)$. There was no significant difference in freedom from reoperation between aortic repair procedures with or without subcommissural root plication (no plication, 96\%; root plication, 98\%; $P=.61)$.

\section{DISCUSSION}

Aortic valve reconstruction has become a new option for the treatment of AR, with an apparently low rate of valverelated complications. ${ }^{5}$ Thus far, attention has mainly focused on technical aspects of repair, but more information is needed about which reconstructive technique is most appropriate for which pathology.

Cusp repair is the primary maneuver in aortic valve repair, and it is mostly applied for prolapse. Cusp prolapse might be an isolated finding in tricuspid aortic valves without concomitant root dilatation. ${ }^{8-15}$ In the current series cusp prolapse as the only cause of regurgitation was seen in $11 \%$ of all patients with AR and 13\% (111/826) of regurgitant aortic valves considered suitable for repair. It was the only cause of regurgitation in $111(22 \%)$ of 504 tricuspid aortic valves.

The pathomechanism of cusp prolapse apparently involves myxomatous degeneration of cusp tissue ${ }^{8-11}$ or distortion of cusp geometry caused by impaired stability in the presence of congenital fenestrations. ${ }^{12-15}$ These fenestrations have been related to root dilatation and increasing age. ${ }^{14,15}$ Our current data indicate that fenestrations are not infrequent in the absence of root dilatation because aortic dilatation by definition was excluded in all patients. Several reports have observed fenestrations in aortic cusps with myxomatous degeneration. ${ }^{8,14}$ On the other hand, we and others have observed these fenestrations in the presence of normal configuration of aortic cusps. In fact, we have seen them in pediatric patients with normal aortic valve function.
Historically, cusp prolapse in tricuspid aortic valves has been corrected by means of paracommissural plication of the free margin, ${ }^{24}$ and limited long-term results have been published. ${ }^{23}$ Investigations on stress distribution of the aortic cusps during diastole provide the explanation for this finding because stress is highest in the paracommissural areas of the free cusp margin. ${ }^{18}$ The central portion of the margin, on the contrary, is exposed to the lowest amount of stress during diastole and thus appears ideally suited for repair maneuvers. ${ }^{18}$

We have previously described that cusp prolapse in bicuspid and tricuspid aortic valves can be corrected by means of plication of the free margin with good midterm stability. ${ }^{21}$ The current report specifically focuses on tricuspid aortic valves, which are geometrically more difficult to repair than bicuspid valves. The presence of fenestrations in conjunction with cusp prolapse poses a problem with a less apparent solution. The presence of fenestrations automatically implies that the free cusp margin consists of thin fibrous strands connecting the central part of the cusp to the commissure. $^{12-15}$ The anatomy easily explains elongation or rupture of the marginal strands as a cause of cusp prolapse because of the altered stress distribution.

Restoration, stabilization, or both of the free cusp margin has been proposed by using thin PTFE sutures in the margin of the affected cusps. ${ }^{17}$ As yet, no published data are available regarding the intraoperative conversion rate, the immediate functional result, and durability. We have not used this technique for tricuspid aortic valves; instead, we have closed the existing tissue defect with a pericardial patch. Autologous pericardium has been shown to be associated with excellent midterm stability. ${ }^{25}$ Closure of the defect eliminates the abnormal stress distribution and thus appears ideal to prevent recurrent cusp deformation after successful repair.

Although the current data do not allow for comparison of different techniques for dealing with fenestrations, the durability indicates that closure of the fenestration leads to a stable result. We cannot conclude whether the use of subcommissural root plication ${ }^{25}$ is a necessary adjunct to this 
form of cusp repair for long-term valve stability. Additional long-term observations will also be necessary to determine the true long-term fate of autologous pericardium inserted into the aortic cusps.

It can be argued that cusps with a tissue defect (ie, fenestrations) are prone to recurrence of deformation and prolapse and therefore should be replaced. Our current experience reaching up to 8 years indicates that this is not the case. Even though the repair is more complex than plication of the free margin, freedom from valve-related complications has been gratifying.

In view of the current result, it might be argued whether all fenestrations encountered during a valve-preserving or valve-reconstructing procedure should be closed. There is no information on the stability that can be expected from a normally configured cusp with fenestrations, and any intervention on the cusp can lead to distortion of the aortic valve. It thus appears reasonable only to address fenestrated cusps that are actually deformed at the time of the operation. Thus far, we have closed up to 3 defects on up to 2 cusps with good results. We have encountered fenestrations in each of the 3 cusps only rarely, and we decided to replace these valves.

In conclusion, cusp prolapse is a relevant cause of AR in tricuspid aortic valves in the presence of normal (ie, nondilated) aortic roots. Prolapse might be due to myxomatous degeneration or elongation or rupture of the marginal strands that border congenital fenestrations. Correction of prolapse can be performed by means of plication of the free margin in the presence of normal cusp tissue and, if present, by closing the fenestration with a pericardial patch. Both techniques lead to equally good midterm results, and the incidence of valve-related complications is low. Repair should be considered as an alternative to replacement in these pathologies.

\section{References}

1. Dujardin KS, Enriquez-Sarano M, Schaff HV, Bailey KR, Seward JB, Tajik AJ. Mortality and morbidity of aortic regurgitation in clinical practice: a long-term follow-up study. Circulation. 1999;99:1851-7.

2. Hammermeister K, Sethi GK, Henderson WG, Grover FL, Oprian C, Rahimtoola SH. Outcomes 15 years after valve replacement with a mechanical versus a bioprosthetic valve: final report of the Veterans Affairs randomized trial. J Am Coll Cardiol. 2000;36:1152-8.

3. Jamieson WR, Burr LH, Miyagishima RT, Germann E, Macnab JS, Stanford E, et al. Carpentier-Edwards supra-annular aortic porcine bioprosthesis: clinical performance over 20 years. J Thorac Cardiovasc Surg. 2005;130:994-1000.
4. Ikonomidis JS, Kratz JM, Crumbley AJ, Stroud MR, Bradley SM, Sade RM, et al. Twenty-year experience with the St. Jude Medical valve prosthesis. J Thorac Cardiovasc Surg. 2003;126:2022-31.

5. Langer F, Aicher D, Kissinger A, Wendler O, Lausberg H, Fries R, et al. Aortic valve repair using a differentiated surgical strategy. Circulation. 2004;110(suppl): II67-73.

6. Cosgrove DM, Rosenkranz ER, Hendren WG, Bartlett JC, Stewart WJ. Valvuloplasty for aortic insufficiency. J Thorac Cardiovasc Surg. 1991;102:571-6.

7. Haydar HS, He GW, Hovaguimian H, McIrvin DM, King DH, Starr A. Valve repair for aortic insufficiency: surgical classification and techniques. Eur J Cardiothorac Surg. 1997;11:258-65.

8. Shapiro LM, Thwaites B, Westgate C, Donaldson R. Prevalence and clinical significance of aortic valve prolapse. Br Heart J. 1985;54:179-83.

9. Tonnemacher D, Reid C, Kawanishi D, Cummings T, Chandrasoma P, McKay CR, et al. Frequency of myxomatous degeneration of the aortic valve as a cause of isolated aortic regurgitation severe enough to warrant aortic valve replacement. Am J Cardiol. 1987;60:1194-6.

10. Agozzino L, de Vivo F, Falco A. de Luca Tupputi Schinosa L, Cotrufo M. Noninflammatory aortic root disease and floppy aortic valve as cause of isolated regurgitation: a clinico-morphologic study. Int J Cardiol. 1994;45:129-34.

11. Allen WM, Matloff JM, Fishbein MC. Myxoid degeneration of the aortic valve and isolated severe aortic regurgitation. Am J Cardiol. 1985;55:439-44.

12. Matthews RJ, Darvill FT. Fenestrations of the aortic valve cusps as a cause of aortic insufficiency and spontaneous aortic valve cusp rupture. Ann Intern Med. 1956; 44:993-1002.

13. Marcus FI, Ronan J, Masanik LF, Ewy GA. Aortic insufficiency secondary to spontaneous rupture of a fenestrated leaflet. Am Heart J. 1963;66:675-8.

14. Kaplan J, Farb A, Carliner NH, Virmani R. Large aortic valve fenestrations producing chronic aortic regurgitation. Am Heart J. 1991;122:1475-7.

15. Akiyama K, Hirota J, Taniyasu N, Maisawa K, Kobayashi Y, Tsuda M. Pathogenetic significance of myxomatous degeneration in fenestration-related massive aortic regurgitation. Circ J. 2004;68:439-43.

16. Carpentier A. Cardiac valve surgery-the "French correction." J Thorac Cardiovasc Surg. 1983;86:323-37.

17. Chiappini B, Pouleur AC, Noirhomme P, Funken JC, Astarci P, Verhelst R, et al. Repair of trileaflet aortic valve prolapse: mid-term outcome in patients with normal aortic root morphology. Interact Cardiovasc Thorac Surg. 2007;6:56-9.

18. Gnynaneshwar R, Kumar RK, Balakrishan KR. Dynamic analysis of the aortic valve using a finite element model. Ann Thorac Surg. 2002;73:1122-9.

19. Swanson M, Clark RE. Dimensions and geometric relationships of the human aortic valve as a function of pressure. Circ Res. 1974;35:871-82.

20. Schäfers HJ, Bierbach B, Aicher D. A new approach to the assessment of aortic cusp geometry. J Thorac Cardiovasc Surg. 2006;132:436-8.

21. Aicher D, Langer F, Adam O, Tscholl D, Lausberg H, Schäfers HJ. Cusp repair in aortic valve reconstruction: does the technique affect stability? J Thorac Cardiovasc Surg. 2007; 134:1533-8.

22. Cabrol C, Cabrol A, Guiraudon G, Bertrand M. Le traitement de l'insuffisance aortique par'annuloplastie aortique. Arch Mal Coeur Vaiss. 1966;59:1305-12.

23. Vahanian A, Baumgartner H, Bax J, Butchart E, Dion R, Fillipatos G, et al. Guidelines on the management of valvular heart disease: the Task Force on the Management of Valvular Heart Disease of the European Society of Cardiology. Eur Heart J. 2007;28:230-68.

24. Trusler GA, Williams WG, Smallhorn JF, Freedom RM. Late results after repair of aortic insufficiency associated with ventricular septal defect. J Thorac Cardiovasc Surg. 1992;103:276-81.

25. Al Halees Z, Al Shahid M, Al Sanei A, Sallehuddin A, Duran C. Up to 16 years follow-up of aortic valve reconstruction with pericardium: a stentless readily available cheap valve? Eur J Cardiothorac Surg. 2005;28:200-5. 